\title{
EFECTIVIDAD DE UN BIOFERTILIZANTE FOLIAR SOBRE EL CULTIVO DE FRIJOL COMÚN (PHASEOLUS VULGARIS), BLUEFIELDS, R.A.A.S.
}

\author{
Yader Mejía Bermúdez ${ }^{[1]}$ \\ Mario Álvarez Arroyo ${ }^{[2]}$ \\ Gladys Luna Bello ${ }^{[3]}$
}

\section{Resumen}

Este trabajo tuvo como propósito evaluar la efectividad de las diferentes dosis de un biofertilizante foliar sobre el rendimiento del cultivo de frijol común (Phaseolus vulgaris) en época de postrera, en la comunidad El Cañal, Kukra River, Bluefields, en el ciclo productivo 2008-2009. Se utilizó un diseño completo al azar, cuatro tratamientos con dos repeticiones, cada repetición constó de 63 plantas de frijol $(\mathrm{n}=126)$. Los tratamientos fueron: T1 (testigo); T2: 0.25 litros de biofertilizante; T3: 0.37 lt. y T4: 0.5 lt. En todos los tratamientos, incluyendo al testigo, se hizo deshierbe y en todos, excepto el testigo, se aplicó el biofertilizante disuelto en 10 litros de agua. Se realizaron tres mediciones $(21,36$ y 51 días) después de la siembra.

Los resultados indican que no existen diferencias significativas entre los tratamientos en la altura de las plantas, pero si en el porcentaje de afectación por herbívoros, así como en el número de vainas por planta y de granos por vaina. El tratamiento $\mathrm{T}_{4}$ registró el menor ataque por herbívoros, la mayor producción en función del número de vainas por planta, de granos por vaina y peso total de los granos de frijol, en cambio el testigo fue el que registró el menor rendimiento con respecto a todas estas variables. El uso del biofertilizante foliar aumentó el rendimiento del cultivo de frijol en todas las dosis.

Palabras claves: abonos orgánicos, rendimiento, trópico húmedo, Nicaragua.

\section{Summary}

The aim of this work was to evaluate the effectiveness of different doses of a foliar biofertilizer on crop production of common bean (Phaseolus vulgaris) throughout season time in the community of El Cañal, Kukra River, Bluefields, during the production cycle 2008-2009. A random design was used, four treatments with two replications, each replication consisted of 63 bean plants $(n=126)$. The treatments

[1] Ingeniero Agroforestal. Graduado en la URACCAN-Bluefields. yameber_o6@yahoo.es

[2] Ingeniero Agroforestal. Graduado en la URACCAN-Bluefields .mogo@yahoo.com

[3] Máster en Recursos Naturales y Desarrollo Rural. Tutora de la investigación. Docente de la URACCAN, Bluefields. glunabello@hotmail.com 
were: $\mathrm{T}_{1}$ (baton), $\mathrm{T}_{2}$ : 0.25 liters of biofertilizer, $\mathrm{T}_{3}: 0.37 \mathrm{lt}$. and $\mathrm{T}_{4}$ : $0.5 \mathrm{lt}$. In all the treatments, including the baton, weeding was done, and in all except the baton, biofertilizer was applied dissolved in 10 liters of water. Three measurements were made (21, 36 and 51 days) after planting.

The results indicate no significant differences between treatments in the height of the plants, but it does in the percentage of affectation by herbivores, as well as in the number of pods per plant and grains per pod. The $\mathrm{T}_{4}$ treatment had the lowest attack by herbivores, the highest production by the number of pods per plant, grains per pod and weight of grains of beans; while the baton registered the lowest production on all these variables. The use of the foliar biofertilizer increased the production of bean crop in all dosages.

Keywords: organic fertilizer, production, humid tropics, Nicaragua.

\section{Introducción}

Uno de los desafíos que enfrentan los agricultores y las agencias de alimentación a nivel mundial, es la necesidad urgente de garantizar la seguridad alimentaria (Luna, 2008). En Nicaragua, la dieta alimenticia está basada en tres granos básicos: frijoles, arroz y maíz (Merino, 1998); Sin embargo, la producción de estos granos ha tendido a disminuir en los últimos años (CASC/Ipade, 2005).

La Costa Atlántica de Nicaragua es una zona con suelos de vocación forestal; no obstante, existen áreas como la zona de Kukra River en la R.A.A.S., que se han caracterizado por producir granos básicos como el frijol. En dicha zona, los productores cultivan este grano, principalmente para el autoconsumo, debido a que obtienen rendimientos muy bajos en las cosechas. De acuerdo con CASC/Ipade (2005), los bajos rendimientos se deben a la influencia de factores como: poca asistencia técnica, mala preparación del terreno, mal manejo de hierbas, densidades de siembra no adecuadas, ataque de plagas y la baja fertilidad del suelo.

Sobre este último factor limitante apuntala esta investigación y para ello se propone evaluar el comportamiento del cultivo de frijol (Phaseolus vulgaris $L$ ) al aplicar un método de fertilización foliar a base de ingredientes naturales y de fácil acceso en las comunidades rurales de la R.A.A.S.; como una manera de dar respuesta a los problemas de baja productividad en esta zona y en otras con características similares.

Esta investigación servirá de base para reorientar las técnicas de manejo y producción que los extensionistas, tanto de ONG's, universidades y proyectos que trabajan en la región y que están sugiriendo a los productores, sobre las dosis y momentos más apropiados para aplicar este tipo de biofertilizantes. 


\section{Metodología}

\section{Área de estudio}

El estudio se realizó en la comunidad El Cañal, zona de Kukra River, a unos 50 km. al suroeste de la ciudad de Bluefields, específicamente en las coordenadas $11^{\circ} 49^{\prime} 18.8^{\prime \prime}$ latitud Norte y $84^{\circ} 00^{\prime} 28.1^{\prime \prime}$ longitud Oeste.

\section{Método}

Inicialmente se realizó un muestreo de suelos para determinar el ph y la textura del mismo. Se tomó una muestra de suelo en cada uno de los tratamientos, y se procedió a medir estas variables haciendo dos repeticiones. Para ello se utilizó un kits portátil.

\section{Diseño experimental}

Se utilizó un Diseño Completamente al Azar (DCA) con cuatro tratamientos y dos repeticiones (cuadro No. 1).

Dentro de cada parcela se establecieron 5 sub-parcela de $125 \mathrm{~m}^{2}$, respetando un efecto de borde de $5 \mathrm{~m}$.

La densidad de siembra fue de 2,946 plantas de frijol en los $625 \mathrm{~m}^{2}$. A todos los tratamientos se le aplicó control de hierbas antes de la aplicación del biofertilizante. El método de siembra fue a través de espeque, depositando de 3 a 4 granos por golpe. El distanciamiento entre surcos, fue de $60 \mathrm{~cm}$ y de $35 \mathrm{~cm}$ entre plantas.

Cuadro No. 1. Tratamientos experimentales, incluyendo al tratamiento control ( $\left.T_{1}\right)$, aplicados al cultivo de frijol.

\begin{tabular}{|c|l|c|c|}
\hline Tratamiento & \multicolumn{1}{|c|}{ Descripción } & Repeticiones & $\begin{array}{c}\text { Aplicaciones de } \\
\text { biofertilizante }\end{array}$ \\
\hline$T_{1}$ & Frijol común + control de maleza. & 2 & 2 \\
\hline$T_{2}$ & $\begin{array}{l}\text { Frijol común + control de malezas + 0.25 } \\
\text { litro de biofertilizante con 10 litros de agua. }\end{array}$ & 2 & 2 \\
\hline$T_{3}$ & $\begin{array}{l}\text { Frijol común + control de malezas + 0.37 } \\
\text { litro de biofertilizante con 10 litros de agua. }\end{array}$ & 2 & 2 \\
\hline$T_{4}$ & $\begin{array}{l}\text { Frijol común + control de malezas + 0.50 } \\
\text { litro de biofertilizante con 10 litros de agua. }\end{array}$ & 2 & 2 \\
\hline
\end{tabular}




\section{Variables}

Se midieron tres variables en un total de $\mathrm{n}=126$ plantas por tratamiento: 1 . Altura de las plantas, 2. Incidencia de herbívoros, además para esta variable se identificó el número de insectos o moluscos promedio de planta por tratamiento, así como el nivel de daño 3. Rendimiento expresada como: número de vainas por planta, número de granos por vaina y peso en libras por tratamiento.

En total se realizaron tres mediciones de estas variables: a los 21, 36 y a los 51 días después de la siembra. Los datos se tomaron después de realizar el control de hierbas y la aplicación del biofertilizante.

Para conocer el rendimiento del cultivo de frijol por tratamiento se realizó el conteo del número de granos por vaina, seleccionando al azar una vaina de cada una de las 63 plantas y se contó el número de semillas de frijol contenidas dentro de cada vaina, luego se estimó el promedio de granos por vaina en cada planta. Finalmente se realizó el pesado en libras del cultivo por cada uno de los tratamientos.

\section{Análisis de datos}

Utilizando el InfoStat (versión 2009) se analizaron pruebas de varianza (anova) con dos repeticiones, para determinar si existen diferencias significativas entre los tratamientos en términos del crecimiento en altura de las plantas de frijol, del daño causado por herbívoros y finalmente del rendimiento del cultivo (vainas por planta y granos por vaina), al aplicar diferentes dosis del biofertilizante. Además, se realizó una prueba post hoc de Tukey para determinar entre cuáles grupos existen diferencias estadísticas. Conjuntamente, se calculó el coeficiente de variación (CV\%), medida de dispersión que indica el grado de variación entre diferentes conjuntos de datos (Gutiérrez, 2000). Para estudios en agronomía, es aceptable hasta un $25 \%$ de CV. Para conocer el número de herbívoros por planta y el rendimiento en libras del cultivo de frijol, se utilizó el programa Excel.

\section{Resultados y discusión}

\section{Análisis de suelo}

Los suelos donde se estableció el experimento son ácidos ya que se ubican en un rango que va de 5.75 a 6 de grado de acidez y la textura de los suelos es franco arcilloso en todos los tratamientos. 


\section{Altura de las plantas}

Los resultados del análisis de varianza indican que no hubo diferencias estadísticas significativas entre los tratamientos, en ninguna de las tres etapas del cultivo de frijol (M1: $\mathrm{p}=0.7564, \mathrm{M}_{2}: \mathrm{p}=0.4729, \mathrm{M}_{3}: \mathrm{p}=0.9840$ ) en términos de la altura de las plantas.

El coeficiente de variación aplicado a cada anova por muestreo es relativamente bajo (5.69\%, 9.75\% y 13.8\%), lo que indica que el tamaño de la muestra es confiable. Ello indica que la variabilidad entre los conjuntos de datos es poca y que las diferencias observadas entre los tratamientos, aunque no fueron estadísticamente significativas, se deben al efecto del tratamiento y no a la influencia de factores externos que podían influir en los resultados (Gutiérrez, 2000).

La prueba de Tukey, muestra que todos los tratamientos o dosis del biofertilizante foliar presentaron medias cercanas entre sí, con respecto a la altura, ubicando a todos estos dentro del mismo grupo A, en los tres momentos del muestreo. (Fig. No. 2)

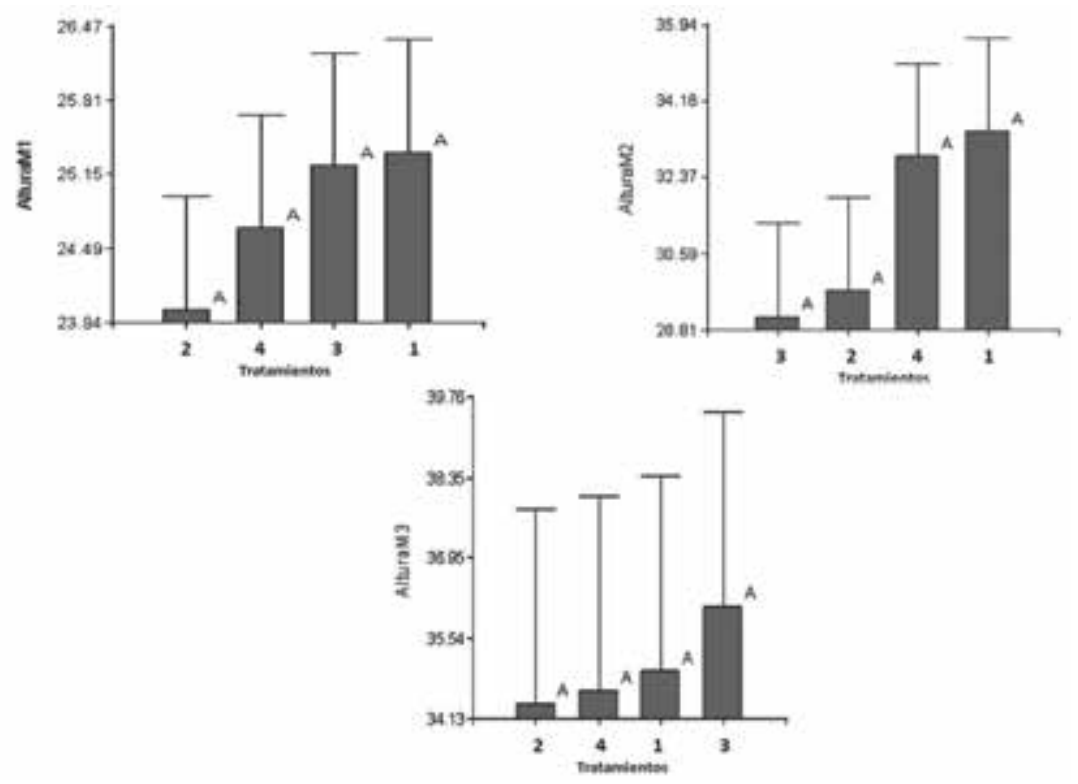

Figura No. 1. Altura de plantas de frijol durante tres muestreos M1: primer muestreo, M2: segundo muestreo y M3: tercer muestreo.

Aunque estadísticamente no existen diferencias entre los tratamientos con respecto al tamaño de las plantas, se puede ver que la altura en algunos tratamientos (ej. tratamiento 3 en el momento 2) si presenta variaciones con respecto a los demás tratamientos. 


\section{Daño de las plantas}

Los resultados del anova indican que en el primer muestreo ( $\left.\mathrm{M}_{1}\right)$ no existen diferencias significativas $(\mathrm{p}=0.2566)$ en el daño causado por herbívoros al cultivo de frijol entre los tratamientos, pero en los siguientes muestreos se presentan diferencias significativas $\mathrm{M}_{2}(\mathrm{p}=0.0010)$ y $\mathrm{M}_{3} \quad(\mathrm{p}=0.004)$, entre los tratamientos. El coeficiente de variación fue alto en el $\mathrm{M}_{1}=38.4$, mientras que en el $\mathrm{M}_{2}=11.42$ y $\mathrm{M}_{3}=15.76$ fue bajo.

Lo anterior puede deberse a que en el primer muestreo el efecto que puede tener el biofertilizante sobre los herbívoros es mínimo, debido al poco tiempo que ha transcurrido después de la aplicación. Mientras que en el M2 y M3, las dosis del biofertilizante (tratamientos) reflejan diferencias significativas entre ellos.

La prueba de Tukey, muestra que el M1 se forma un solo grupo (A); sin embargo en el $\mathrm{M}_{2}$ se forman dos grupos: $\mathrm{B}$ ( $\mathrm{T}_{1} \mathrm{y} \mathrm{T}_{3}$ ), que fueron los tratamientos que registraron mayores daños por herbivoría y $\mathrm{A}_{(} \mathrm{T}_{2}$ y $\left.\mathrm{T}_{4}\right)$ con menores registros de daño foliar.

En el M3 también se forman dos grupos, pero esta vez el grupo A está formado por tres tratamientos $\left(\mathrm{T}_{4}, \mathrm{~T}_{2}\right.$ y $\left.\mathrm{T}_{3}\right)$ los cuales presentan los menores daños por herbivoría, y en el $\mathrm{B}$ sólo se encuentra el $\mathrm{T} 1$, siendo esté el que registró mayor daño por herbívoria.

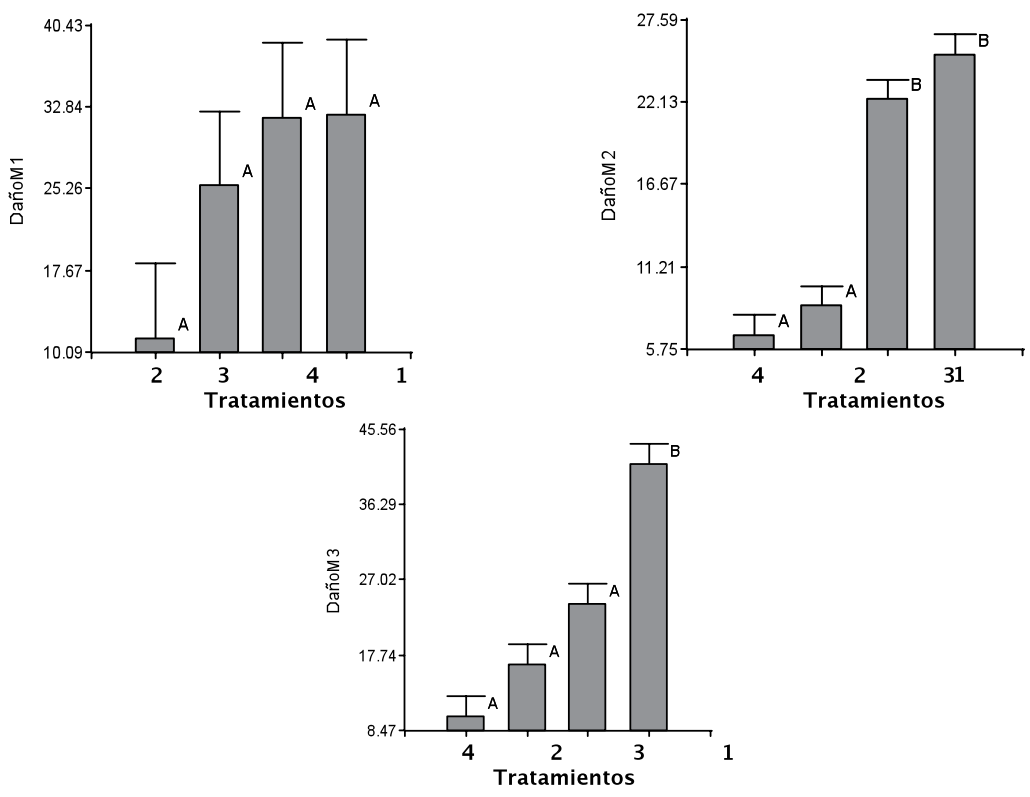

Figura No. 2. Daño de plantas de frijol durante tres muestreos M1: primer muestreo, M2: segundo muestreo y M3: tercer muestreo. 
Empero, es necesario resaltar que todas las dosis del biofertilizante tuvieron un efecto sobre el control de los herbívoros después los 36 días de establecido el cultivo.

Por otro lado, al relacionar la variable crecimiento con el porcentaje de afectación, los resultados indican que los herbívoros al parecer no tuvieron un efecto significativo en el crecimiento de las plantas de frijol, a pesar que el tratamiento T1 (testigo) presentó el mayor porcentaje de daño en el tercer muestreo, también fue uno de los que mostró mayores alturas. Contrariamente a lo esperado, las plantas con menor porcentaje de afectación por herbívoros no crecieron más que las plantas agredidas por estos. Figueroa y Castro (2000), explican que probablemente existen mecanismos de compensación que permiten a las plantas agredidas crecer tanto como las plántulas poco afectadas por los herbívoros. Algún mecanismo podría reasignar los nutrientes de las plántulas hacia la parte aérea para compensar la pérdida de tejido foliar.

\section{Presencia de herbívoros}

El tratamiento T1 fue el que presentó mayor número de herbívoros durante el ciclo del cultivo (69 individuos), lo cual se corresponde con los resultados del porcentaje de afectación, que indican que las plantas de este tratamiento también fueron las que registraron el mayor daño foliar en el segundo y tercer muestreo.

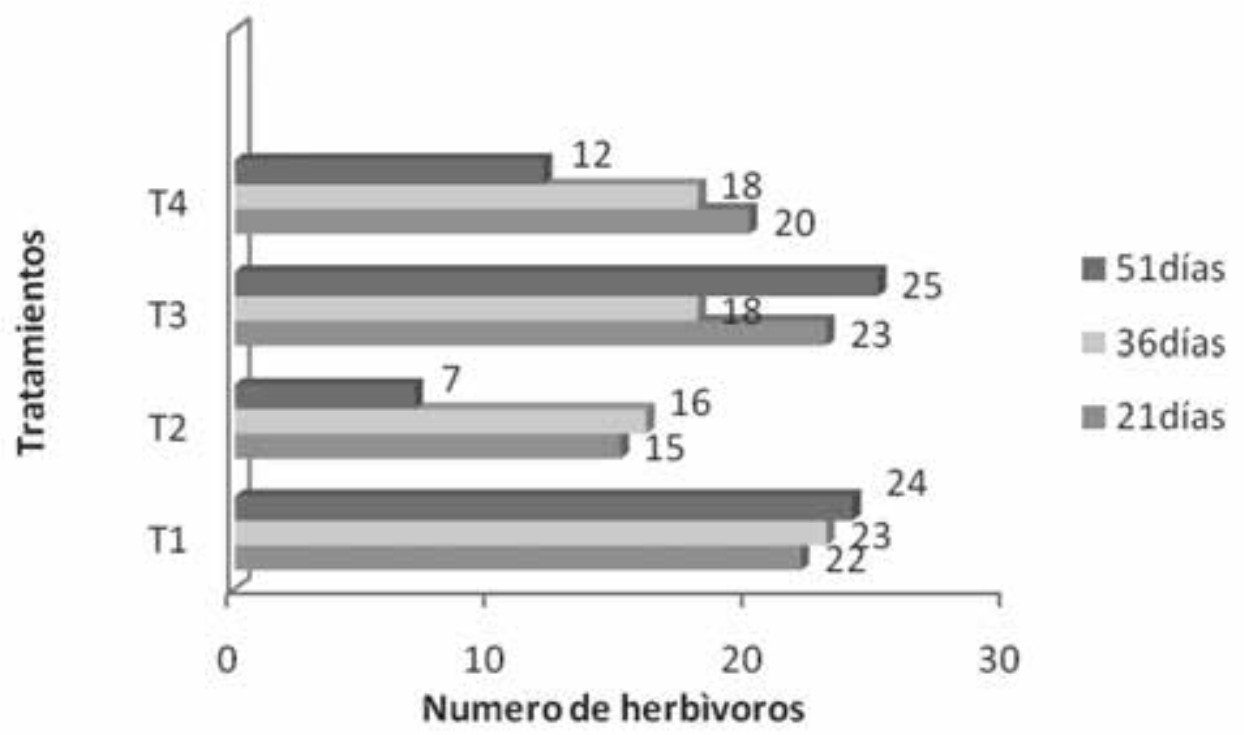

Figura No. 3. Presencia de herbívoros en el cultivo de frijol. 
Por otro lado, al comparar estos resultados con los datos de crecimiento en altura de las plantas se observa que este tratamiento es el que registró una de las mayores alturas y una mayor presencia de herbívoros.

En cambio el tratamiento $\mathrm{T}_{4}$, fue uno de los que registraron el menor número de herbívoros (51 individuos) y presentó el menor daño foliar durante los dos últimos muestreos. Además, por observaciones de campo, se encontró que este tratamiento no creció en altura, pero si en la extensión de su follaje (ramificación de la planta), lo cual indica que al haber mayor disponibilidad de alimento (hojas) los herbívoros son mas atraídos (Bernal, et al. 1998), demostrando con ello que el biofertilizante además de abonar la planta, también puede actuar como repelente para los herbívoros que atacan el cultivo.

\section{Rendimiento}

\section{Número de vainas por planta}

Existen diferencias estadísticas significativas $(\mathrm{p}=0.0143)$ en el número de vainas por plantas entre los tratamientos.

El coeficiente de variación fue bueno (12.24) indicando que los resultados se deben al efecto del tratamiento y no a factores externos.

La prueba de Tukey indica que los tratamientos $\mathrm{T}_{1}$ y $\mathrm{T} 2$ pertenecen al mismo grupo (A), mientras que los tratamientos $\mathrm{T}_{3} \mathrm{y}_{4}$ forman grupos independientes ( $\mathrm{AB}$ y B) respectivamente; resultados interesantes ya que la formación de tres grupos independientes indica que los tratamientos tienen un mayor efecto sobre la producción de vainas en el cultivo.

Según el conteo de vainas por planta el tratamiento $\mathrm{T}_{4}$ es el que presenta mayor rendimiento en cuanto a la producción promedia de vainas (18.67), mientras que el tratamiento $\mathrm{T}_{1}$ (testigo) fue el que presentó la menor producción de vainas por planta (8.89), lo que evidencia que una mayor dosis de biofertilizante $\left(\mathrm{T}_{4}=\right.$ o.5lts) estimuló una mayor producción de vainas en las plantas, lo que indica que los nutrientes que ofrece dicho fertilizante son asimilados exitosamente por la planta.

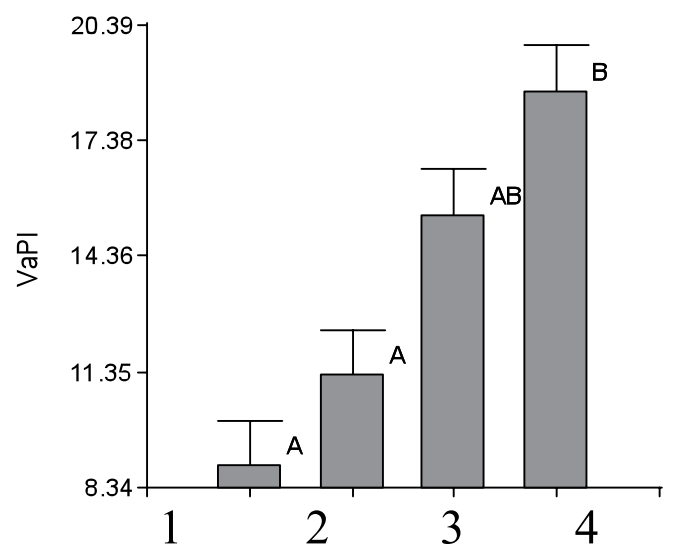

Figura No. 4. Número de vainas por planta. 


\section{Granos por vaina en el tratamiento}

El análisis de varianza indica que hubo diferencia estadísticamente significativas $(\mathrm{p}=0.0276)$ entre los tratamientos, con respecto al número de granos por vaina por planta, entre tratamientos. El coeficiente de variación fue muy bueno (1.40).

Los tratamientos formaron tres grupos según la prueba de Tukey, un grupo $\mathrm{A}$ integrado por el $\mathrm{T}_{2}$, grupo $\mathrm{AB}$ integrado por $\mathrm{T}_{3} \mathrm{y} \mathrm{T}_{1} \mathrm{y}$ un grupo $\mathrm{B}$ compuesto por $\mathrm{T}_{4}$; siendo este último el que presentó el mayor número de granos por vainas y el $\mathrm{T}_{2} \mathrm{el}$ que registró la menor cantidad de granos por vaina. Es importante resaltar que el tratamiento $\left(\mathrm{T}_{4}\right)$ es el que presentó la menor afectación por herbívoros, la mayor producción de vainas por plantas, y también la mayor producción de granos por vaina. Lo que sugiere que el biofertilizante, promueve la salud de la planta y por ello, una planta mejor nutrida es más resistente al ataque de herbívoros y consecuentemente tendrá mayor éxito reproductivo en flores y frutos. (Morales, et al. 2001).

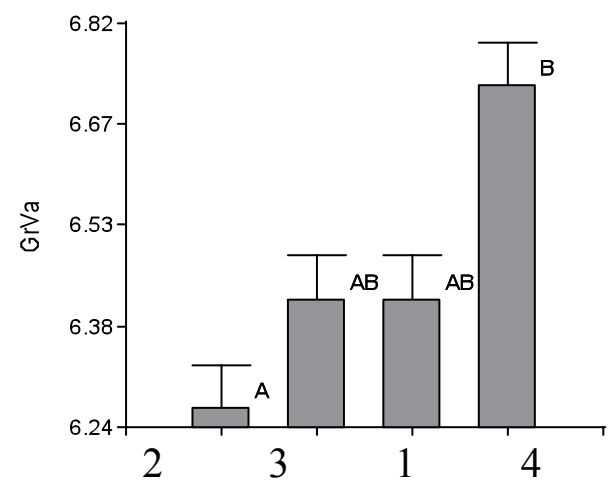

Figura No. 5. Granos por vaina por tratamiento.

Peso de los granos de frijol por tratamiento

El peso de los granos de frijol común, muestra un comportamiento variado entre tratamientos.

Cuadro No. 2. Rendimiento en Peso y porcentaje del cultivo de frijol en diferentes tratamientos.

\begin{tabular}{|c|c|c|c|}
\hline \multirow{2}{*}{ Tratamiento } & \multicolumn{2}{|c|}{ Rendimiento por Área } & \multirow{2}{*}{ Porcentaje\% } \\
\cline { 2 - 3 } & Ibs/625 $\mathbf{m}^{\mathbf{2}}$ & $\mathbf{q q} / \mathbf{h a}$ & \\
\hline $\mathrm{T}_{1}$ & 33 & 5.28 & 15 \\
\hline $\mathrm{T}_{2}$ & 48 & 7.68 & 21.8 \\
\hline $\mathrm{T}_{3}$ & 59 & 9.44 & 26.8 \\
\hline $\mathrm{T}_{4}$ & 80 & 12.80 & 36.4 \\
\hline Total & 220 & 35.2 & 100 \\
\hline
\end{tabular}

Nota: Cada parcela del tratamiento tenía un área de $25 \times 25 \mathrm{~m}$. 
El tratamiento $\mathrm{T}_{4}$ promedió 12.8 qq por ha., resultado excelente, ya que Centeno y Plazaola (2007), en un diagnóstico socio-económico sobre los rendimientos de este cultivo en la zona reportaron $6.96 \mathrm{qq} / \mathrm{ha}(4.37 \mathrm{qq} / \mathrm{mz})$, dato superado en un $45.6 \%$ por el T4. El T1 (testigo) por su parte, alcanzó 5.28qq/ha, rendimiento ligeramente inferior a lo reportado por Centeno y Plazaola (2007). El resto de los tratamientos ( $\mathrm{T}_{2}$ y $\mathrm{T}_{3}$ ) también ostentan rendimientos superiores a los de estos autores, lo que demuestra las ventajas de este sistema de fertilización (en cualquiera de las dosis) sobre el testigo, para las condiciones estudiadas.

Los rendimientos obtenidos en los tratamientos con biofertilizante se pueden explicar por la fertilización orgánica utilizada, lo que hace que la planta disponga de todos los nutrientes en la zona donde se encuentran las raíces, favoreciendo la elevación de los rendimientos (Gómez et al., 2008).

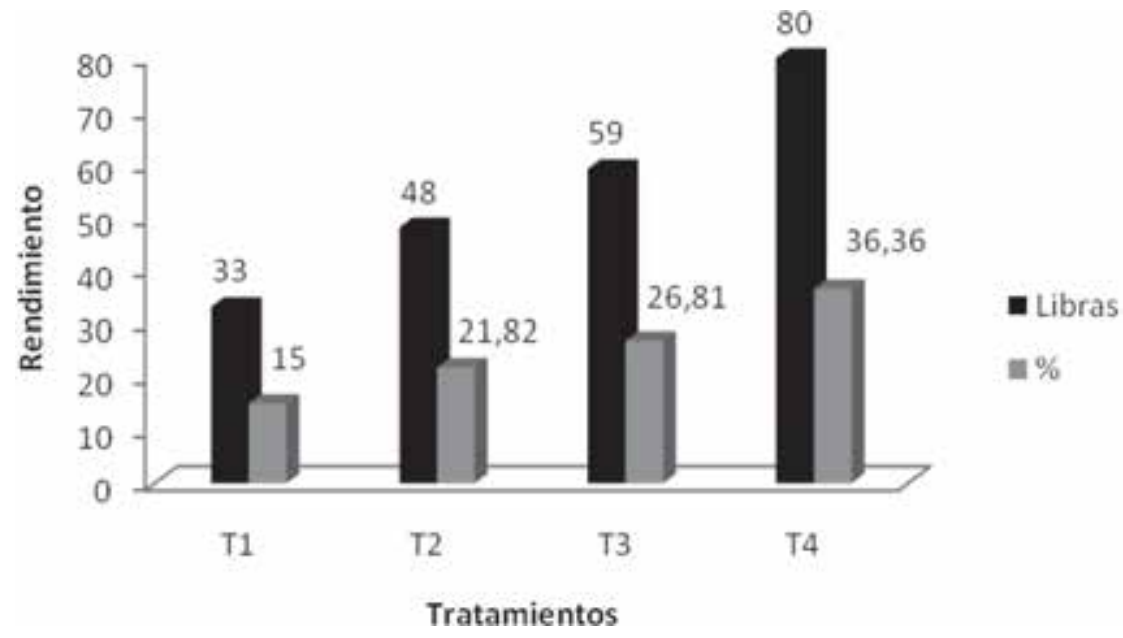

Figura No.6. Peso de los granos de frijol por tratamiento.

Además de la fertilización, también la historia del suelo en el área de cultivo pudo influir en los resultados, pues es un área que se ha utilizado únicamente para sembrar frijoles durante un ciclo productivo y se deja en descanso por tres años, lo que permite que los nutrientes del suelo se recuperen. Por otro lado, el deshierbe realizado durante los momentos iniciales, pudo evitar que la competencia con las arvenses fuera alta, facilitando su establecimiento y buenos rendimientos.

Estos resultados coinciden con los obtenidos por otros autores en condiciones tropicales, en diferentes cultivos donde se aplicaron fertilizantes orgánicos. Bernal et al. (1998) incrementaron los rendimientos agrícolas cuando se aplicó composta en la fase de crecimiento del cultivo de frijol. Gómez et al. (2008), mejoraron significativamente el rendimiento de frijol y de rábano en condiciones tropicales al aplicar técnicas de manejo y fertilización del cultivo. Al evaluar los efectos del biofertilizante en este 
estudio, se observó que el método de fertilización tiene resultados positivos, lo cual indica el potencial de esta tecnología para incrementar los rendimientos del cultivo en condiciones del productor rural.

\section{Conclusiones}

Los resultados indican que la aplicación del biofertilizante foliar fue efectivo en función del rendimiento (vainas/planta, granos/vaina y peso) para todos los tratamientos, en comparación con el testigo, por tal razón se considera que es una alternativa para maximizar los rendimientos del cultivo de frijol en la comunidad El Cañal, en la zona de Kukra River.

Los tratamientos con aplicación de biofertilizante foliar presentaron un menor porcentaje de afectación por herbívoros en su follaje, lo que sugiere que el biofertilizante funciona como repelente natural efectivo, sobre todo en etapas avanzadas del cultivo (segunda y tercera aplicación). Sin embargo, no se registran mejores resultados en altura entre los tratamientos con y sin biofertilizantes.

El tratamiento $\mathrm{T}_{4}$ registró el menor ataque por herbívoros, la mayor producción en función del número de vainas por planta, de granos por vaina y peso total de los granos de frijol, lo que sugiere que esta es la dosis ( $1 / 2$ litro de biofertilizante disueltos en 10 lts. de agua) que registra los mejores rendimientos en el sitio de estudio, por tanto la más recomendable para la zona. No obstante, es importante aclarar que mayores dosis a ésta no necesariamente representan mayor rendimiento del cultivo, ello requerirá de más investigaciones.

Por el contrario, el tratamiento T1 (testigo) arroja la mayor incidencia de plagas, el menor rendimiento del cultivo (en todas las variables medidas), a pesar de haberse comportado como uno de los tratamientos con las mejores alturas durante todo el ciclo del cultivo, lo cual, según la literatura, pudo haber sido una de las razones para ser más atractivo por la disponibilidad de alimento para el herbívoro, lo que determinó el bajo rendimiento.

El tratamiento $\mathrm{T}_{3}$ mostró un comportamiento interesante, ya que el daño por herbívoros fue alto en el segundo y tercer muestreo en este tratamiento; empero, el rendimiento en vainas por planta fue alto, cercano al $\mathrm{T}_{4} \mathrm{y}$ el número de granos por vaina no fue diferente al $\mathrm{T}_{1}$; lo que sugiere que los herbívoros solo afectaron el follaje, pero no la producción de vainas, y por tanto el rendimiento en peso fue alto. Lo que indica que los herbívoros no son una plaga en este cultivo.

El rendimiento del cultivo de frijol fue mayor en este estudio, en comparación con los registros de la producción tradicional en esta zona, demostrándose con esto la validez del sistema de fertilización ( $\mathrm{T}_{4}$ ) en el cultivo de frijol como monocultivo. 


\section{Recomendaciones}

Dados los hallazgos importantes en este estudio, y por ser uno de los primeros en medir diferentes dosis del biofertilizante foliar se sugiere repetir el ensayo utilizando un mayor número de réplicas.

Realizar estudios de los factores químicos del suelo antes del cultivo y después de este, así como del biofertilizante a aplicar.

Estudios que detallen el comportamiento de las poblaciones de herbívoros en las diferentes fases del cultivo y en todas las etapas de crecimiento del insecto (huevos, larvas y adultos) y relacionarlo con los mejores tiempos y dosis de aplicación de métodos de control.

Para los productores de la zona de Kukra River se recomienda hacer uso de las dosis del $\mathrm{T}_{4}$ (o $\left.\mathrm{T}_{3}\right)$, ya que es de bajo costo e incrementan los rendimientos del cultivo de frijol.

\section{Lista de referencia}

Bernal M.P., Navarro, A, Sánchez, M, Roig, A. Cegarra, J. (1998). The influence of sewage sludge component stability and maturity on carbon and nitrogen mineralization. Soil Biol. Biochem. 30 (3):305-313.

CASC/ IPADE. (2005). Informe de desarrollo Humano. Las Regiones Autónomas de la Costa Caribe de Nicaragua.

Centeno, I.; Plazaola, R. (2007). Factores que inciden en la rentabilidad productiva de los cultivos Maíz (Zea Mays) y Frijol (Phaseolus vulgaris) en la comunidad de las Breñas zona de Kukra River durante el ciclo productivo 2005 - 2006. Monografía URACCAN.

Gutiérrez Espeleta E. (2000). Métodos estadísticos para las ciencias biológicas. Primera Edición, primera reimpresión. EUNA. Heredia, Costa Rica. Pag.6-7

Franco, J. (2001). Manual de Ecología. Trillas Casa editorial México.

Figueroa \& Castro (2000). Efecto de herbivoros y patógenos en la sobrevivencia y crecimiento de plántulas en un fragmento del bosque templado húmedo de Chiloé, Chile. Revista Chilena de Historia Natural 73: 163-173. 
Luna, G. (2008). Conocimiento local Ulwa: Insectos y Prácticas Agrícolas en la Región Autónoma Atlántico Sur, Nicaragua. Tesis de Maestría. Chiapas, México.

Merino, Gerardo. (1998). Evaluación de los impactos del cambio climático en la seguridad alimentaria de El Salvador. Ministerio de Medio Ambiente y Recursos Naturales. Primera Comunicación Nacional sobre Cambio Climático. San Salvador, El Salvador. Pp. 16-

Morales, H., Perfecto I., y Ferguson, B. (2001). Traditional Cakchiquel soil fertilization and its impact on insect pest populations in corn. Agriculture, Ecosystems and Environment 84:145-155.

Maes, J.M. (1999). Insectos de Nicaragua. Catálogo de los Insectos y Artrópodos Terrestres de Nicaragua Volumen II. Ministerio Agropecuario y Forestal, CEDOC, Managua, Nicaragua.

Acción Medica Cristiana (A.M.C) (2007). Kukra River, Managua, Nicaragua. En línea: http://www.amc.org.ni/anterior/territoriokukrariver.html

Colprocah (2004). Manejo Integrado de la Babosa. Tegucigalpa, M.D.C., Honduras C.A. En línea: http://www.colprocah.com/secciones/agricultura/ManejoPlagas/ babosa

Gómez, M.; Sierra, M.; González, M.; Cantú, M.; Ramírez, A.; Wong, J.; Manjarrez, M.; \& Ramírez, J. (2008). Hibrido de Maíz de alto rendimiento para el trópico húmedo y seco de México. Instituto Nacional de investigaciones forestales, Agrícolas y pecuarias Texcoco, México. En línea: http://redalyc.uaemex.mx/ pdf/608/60834112.pdf

M. García. s/f. Uso de biofertilizantes en el cultivo del frijol (Phaseolus vulgaris L) en suelos arenosos. En línea: http://www.monografias.com/trabajos55/biofertilizantefrijol-suelo- arenoso/biofertilizante-frijol-suelo-arenoso.shtml 